\title{
Optical effects of wakefields in the PEP-II Stanford Linear Accelerator Center B Factory
}

\author{
S. Heifets, S. Novokhatski, and D. Teytelman \\ Stanford Linear Accelerator Center, Stanford University, Stanford, California 94309, USA
}

(Received 18 September 2006; published 9 January 2007)

\begin{abstract}
Wakefields defining beam stability affect also the beam optics and beam properties in high current machines. In this paper we present observations and analysis of the optical effects in the PEP-II Stanford Linear Accelerator Center B Factory, which has the record in achievement of high electron and positron currents. We study the synchronous phase and the bunch length variation along the train of bunches, overall bunch lengthening, and effects of the wakes on the tune and on the Twiss parameters. This analysis is being used in upgrades of PEP-II and may be applied to future B factories and damping rings for linear colliders.
\end{abstract}

DOI: 10.1103/PhysRevSTAB.10.011001

\section{INTRODUCTION}

The wakefields generated by the beam in the beam pipe are recognized as the cause of the beam instabilities in the high current machines [1]. The wakefields are also the cause of numerous optical effects which do not lead to bunch instability but may be important for optimization of the beam dynamics and luminosity of a collider. Quite often the optics of a machine is studied and optimized at low currents. However, the tune and Twiss parameters vary with current, and the optics at collision can differ from the optimized optics at low current. Therefore optimization of optics at low currents does not necessarily mean optimization of the luminosity of a collider. For example, the optical model developed for PEP-II is in quite good agreement with measurements at low currents but failed to give quantitative agreement in the high current regime [2]. More than that, in the multibunch machines the wakes affect different bunches differently. Some such effects were observed and discussed in the context of the harmonic rf systems [3,4] but still colliders with conventional rf systems deserve more detailed analysis at higher currents. The second reason is given by the need for study of the machine impedance [5]. There are three main contributions to the impedance budget: the rf cavities, resistive wall (RW) impedance, and the impedance generated by small vacuum components of the ring such as bellows, beam position monitors, tapers, masks, vacuum ports, etc. The first two components give long-range wakes and are responsible for coupled-bunch (CB) instabilities, longitudinal and transverse, respectively. Usually, the impedance of these components is well known from cold measurements of rf cavities, 3D simulations of EM fields of the cavities, and the machine geometry. It is much more difficult to determine the impedance of vacuum components both numerically and experimentally. Different methods were used to infer the machine impedance measuring the closed orbit variation with current [6], betatron phase advance [7], the tune variation and the head-tail damping [8], the synchronous phase, the bunch length, and the betatron cou-
PACS numbers: 29.27.Bd, 29.20.Dh, 41.60.- m, 52.59.-f

pling [9]. Not all methods are equally effective for large and carefully designed machines with low impedance such as PEP-II. In our experience, analysis of the current dependence of the tunes, measurements of the synchronous phase, and the bunch length were crucial to extract impedance parameters from the measurements.

In this paper, we analyze some of the current dependent effects. Results are obtained for PEP-II low energy ring (LER) but may be relevant for other projects such as Super B factories [10] and the ILC project. In Appendix A we discuss in detail the relation between the rms of a single bunch and the bunch in a train of bunches. We summarize our findings in the conclusion.

\section{VARIATION OF THE BUNCH LENGTH AND SYNCHRONOUS PHASE ALONG THE TRAIN OF BUNCHES}

We used beam spectra for the bunch length measurement [11]. To resolve bunch length along the bunch train, we used a spectrum analyzer in the gated regime. Results of measurements in the LER of PEP-II depicted in Fig. 1

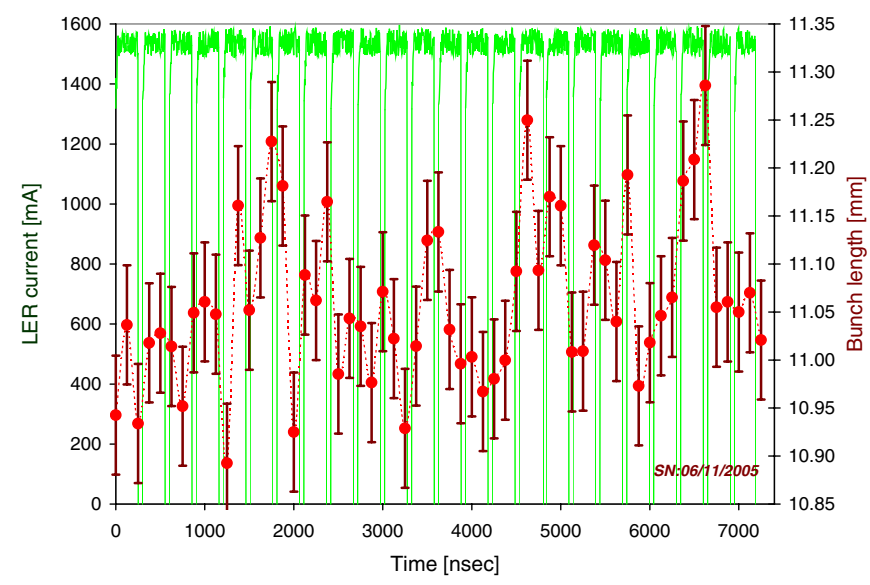

FIG. 1. (Color) Variation of the bunch length (red) and the bunch current (green) along the 24 minitrains of the LER current. Total number of bunch is 1440 . Total current is $2.2 \mathrm{~A}$. 
show variation of the bunch length along the 24 minitrains of the LER current of 2.2 A. Total number of bunches is 1440.

This variation is caused by the gaps between minitrains and one large "ion" gap [12]. The ion gap in the LER matches the ion gap in the electron high energy ring (HER) and is needed also for the beam abort due to the finite rise time of a kicker. In the last runs, the machine is filled with 1722 bunches with the bunch separation $2 \lambda_{\mathrm{rf}}$ and $2 \%$ gap. One can expect that a gap with the length $s_{g}$ would generate transients of the rf voltage of the order of

$$
1-e^{-\left(\omega_{r f} s_{g}\right) /\left(2 Q_{L} c\right)} \text {. }
$$

Effect, actually, is larger. A small $2 \%$ gap gives substantial variation of the wake affecting different bunches and, as result, of the synchronous phase along the train. The variation of the synchronous phase contributes to variation of the slope of the rf voltage and, therefore, causes variation of the synchronous frequency $f_{s}$. An example of such variation calculated for LER parameters at the total $\mathrm{rf}$ voltage $V_{\text {tot }}=4.5 \mathrm{MV}$ is shown in Fig. 2. The variations of the synchronous phase for the PEP-II with large beam loading is due, mostly, to the fundamental mode of the rf cavities. Other rf higher order modes give a smaller contribution to this effect.
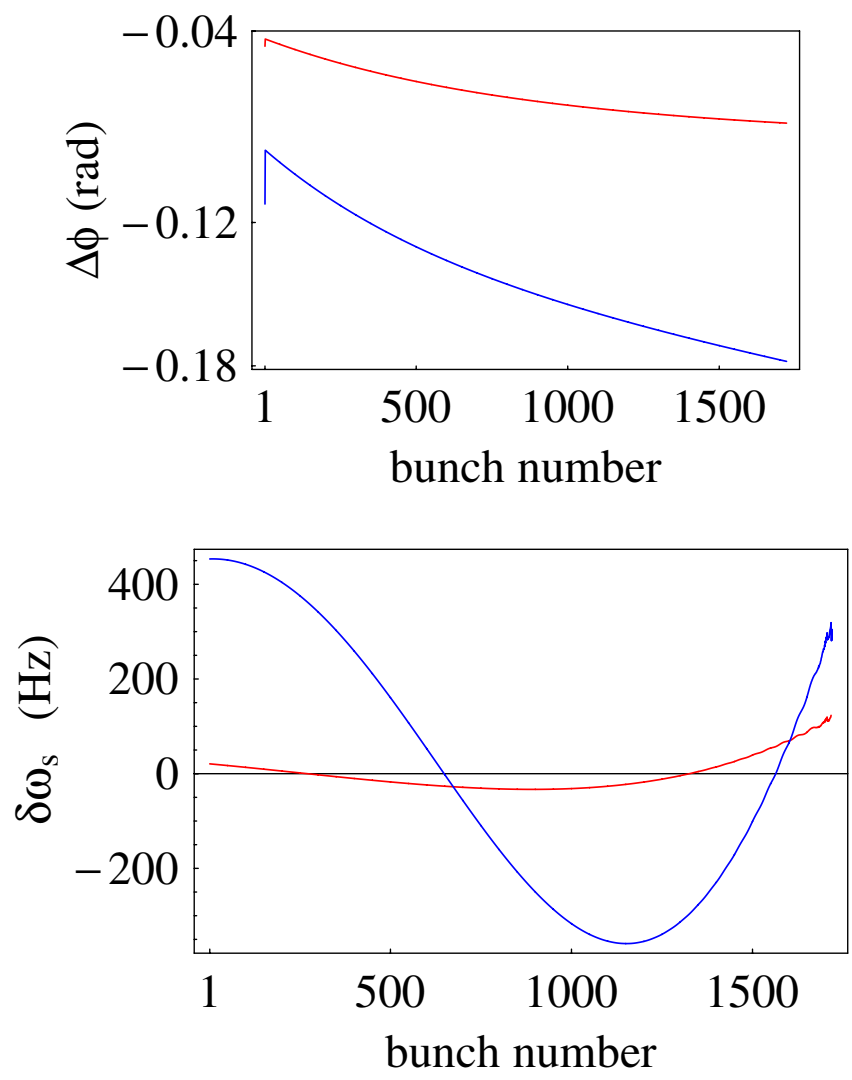

FIG. 2. (Color) Synchronous phase (top) and synchrotron frequency (bottom) along the train caused by the ion gap for the beam current 0.5 (red line) and 1.0 Amp (blue line).

\section{A. Bunch length variation}

Transient variation of the rf phase and the synchrotron frequency of the bunches along the train affect the rms length of a bunch, see discussion in Appendix A. Results of calculations are shown in Fig. 3, where the bottom plot gives variation of the bunch length caused by the transients of the rf voltage, and the upper plot includes both the transients and the bunch lengthening due to potential well distortion (PWD). The amplitude and the period of the variation depend on the beam current $I_{B}$ increasing with $I_{B}$. Both, the amplitude and the modulation frequency, depend on the detuning of the cavity and the effect is maximum when the detuning is equal to the revolution frequency which takes place for PEP-II parameters at, approximately, $I_{B}=2.5 \mathrm{~A}$.

The bunch length measured without gating on individual bunches gives the average rms over the whole train. The averaging over the train rms gives current dependence shown in Fig. 4. Calculations are based on the estimate of the LER impedance [13] which gives inductance of the vacuum components $L_{\text {ind }}=80 \mathrm{nH}$. The number is, certainly, only an estimate. The real inductance is, probably,
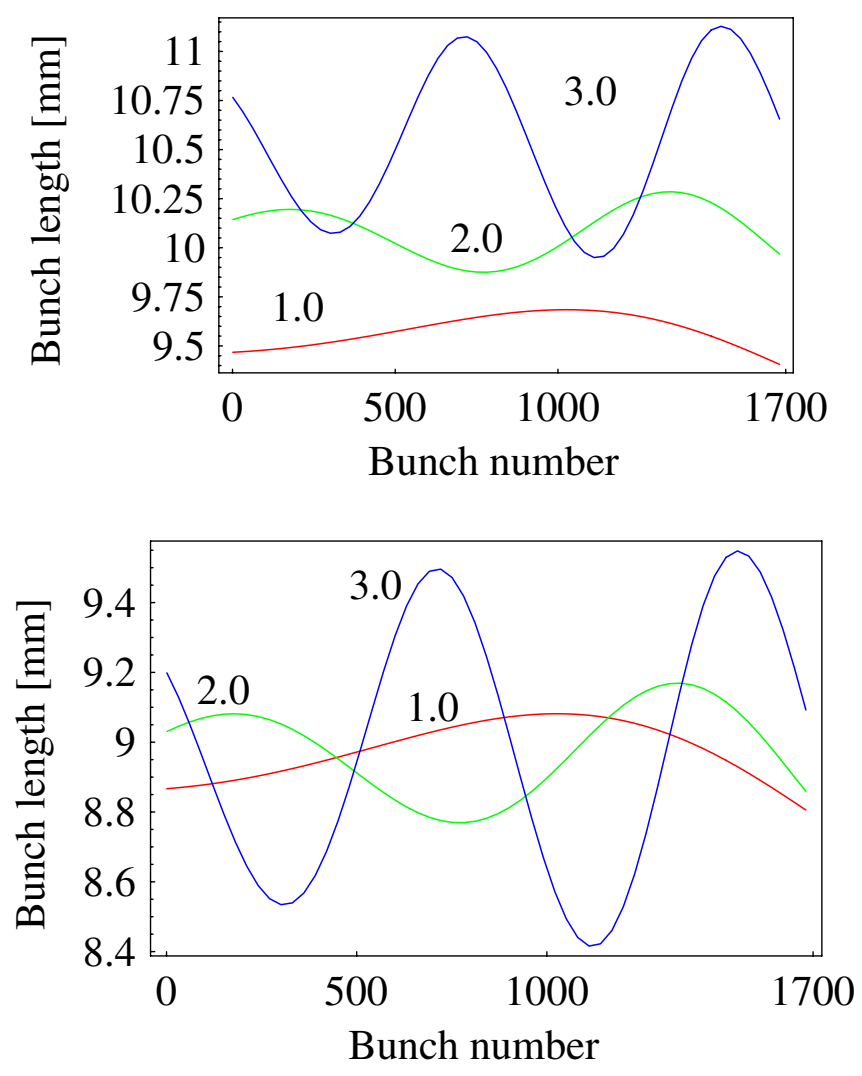

FIG. 3. (Color) Calculated variation of the bunch length along the train for LER $V=4.5 \mathrm{MV}$ and the beam currents (indicated in the figure) $I_{B}=1.0,2.0$, and $I_{B}=3.0$ A. Bottom plot: results of only gap transients. Upper plot: variation due to the gap transients and the PWD. Note the increase of the variation with the detuning. 


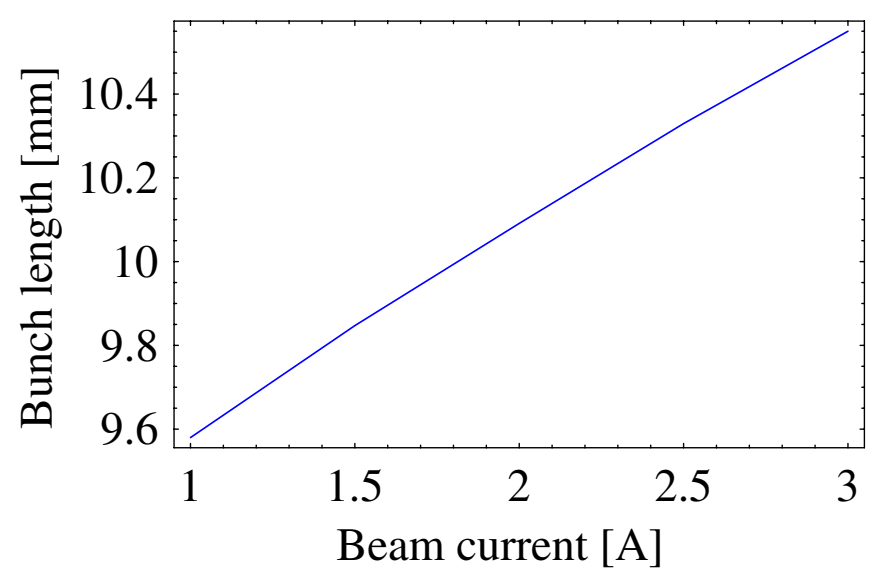

FIG. 4. (Color) Averaged over the train bunch length vs beam current. Inductance $L=80 \mathrm{nH}, d \sigma / d I_{B}=0.83 \mathrm{~mm} / \mathrm{mA}$. Radio-frequency voltage is $4.5 \mathrm{MV}$.

higher partially due to vacuum components added after the estimate was carried out. The fit gives

$$
\frac{d \sigma_{l}}{I_{B}}=0.83 \mathrm{~mm} / \mathrm{mA}
$$

The measurements in PEP-II give close numbers: the streak-camera measurements give $d \sigma / d I_{B}=0.8 \mathrm{~mm} / \mathrm{mA}$ while the bunch length extracted from the bunch spectrum gives somewhat higher value, $d \sigma / d I_{B}=1.45 \mathrm{~mm} / \mathrm{mA}$. It is encouraging that calculations give a result close to the measurements although it is not clear whether the remaining discrepancy is due to underestimated inductance in calculations or due to errors of measurements.

It is worth making the following remarks. Generally, the bunch length for a single bunch in the ring $\sigma_{1}$ and the average bunch length in a train of bunches $\sigma$ are different for the same rf voltage and the bunch current. It can be shown (see Appendix A) that, in the linear over the beam current approximation, they are related:

$$
\sigma_{1}^{2}=\left(\frac{\hat{\omega}_{s}}{\omega_{s, 0}}\right)^{2} \sigma^{2}
$$

where $\omega_{s, 0}$ is the zero-current synchrotron frequency and $\hat{\omega}_{s}$ takes into account the short- and the long-range wakefields,

$$
\hat{\omega}_{s}^{2}=\omega_{s, 0}^{2}+\lambda c^{2}\left\langle W^{\prime}\right\rangle .
$$

More discussion is given in Appendix A.

It is worth noting, that $\omega_{s}$ measured by different methods can give different results. The best method of determining $\omega_{s}$ is by measuring the frequency of the second synchrotron sideband of the rf frequency which is less affected by the feedback system. The actual situation is quite complicated because each bunch centroid oscillates with different frequencies. Fortunately, the spread of CB frequencies and is not large.

As it is well known, the head-tail instability can give the shift of the synchrobetatron sideband but should not be

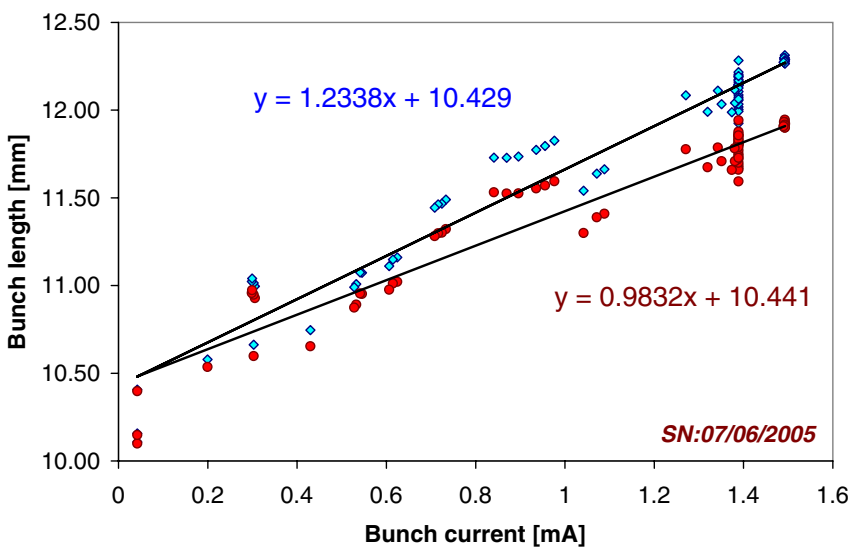

FIG. 5. (Color) The measured bunch length in a train of bunches (blue) and the bunch length of a bunch with the same rf voltage and bunch current calculated from the measured synchrotron tune (red).

included in Haissinski calculations because the shift is the result of the $x-z$ correlation in a bunch and does not change the longitudinal oscillations of the bunch centroid. The $2 \omega_{s}$ sideband give better measurements of $\omega_{s}$ but, actually, there are two $2 \omega_{s}$ lines in the spectrum. One is due to the nonlinearity of the oscillations of the bunch centroid, another one is the coherent quadrupole longitudinal CB oscillations of the bunch profile. These frequencies are, generally, different.

Equations (2) and (3) can be verified experimentally. Comparing with experiment, we have to remember that both potential well distortion and the synchrotron frequency change with current. The first effect can be defined keeping the synchrotron frequency fixed. Figure 5 show two lines: one (blue) is direct measurement of the bunch length in a train of bunches as a function of beam current. Another one (red) depicts variation of the bunch rms length at fixed synchrotron frequency calculated using measured frequency of synchrotron oscillations.

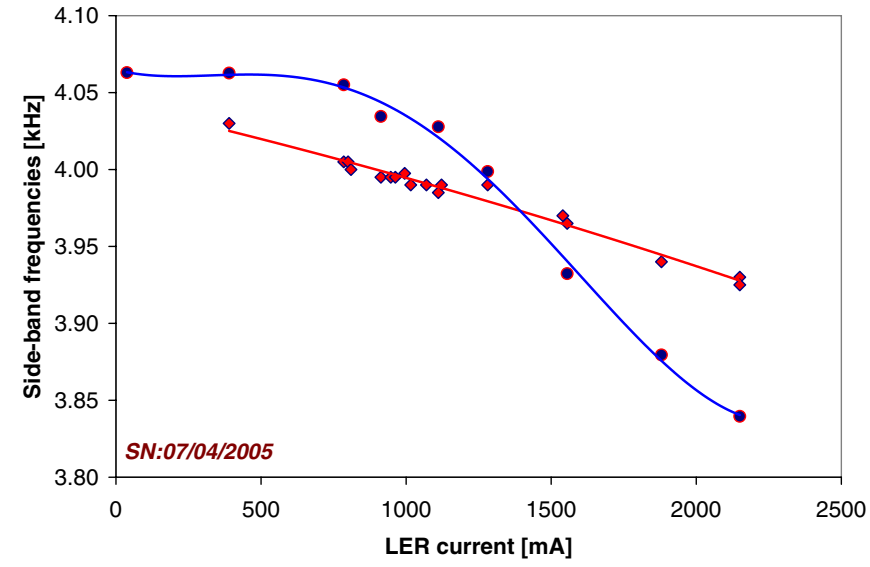

FIG. 6. (Color) Synchrotron frequency measured from the first (blue) and the second sideband (red) in the beam spectrum. 


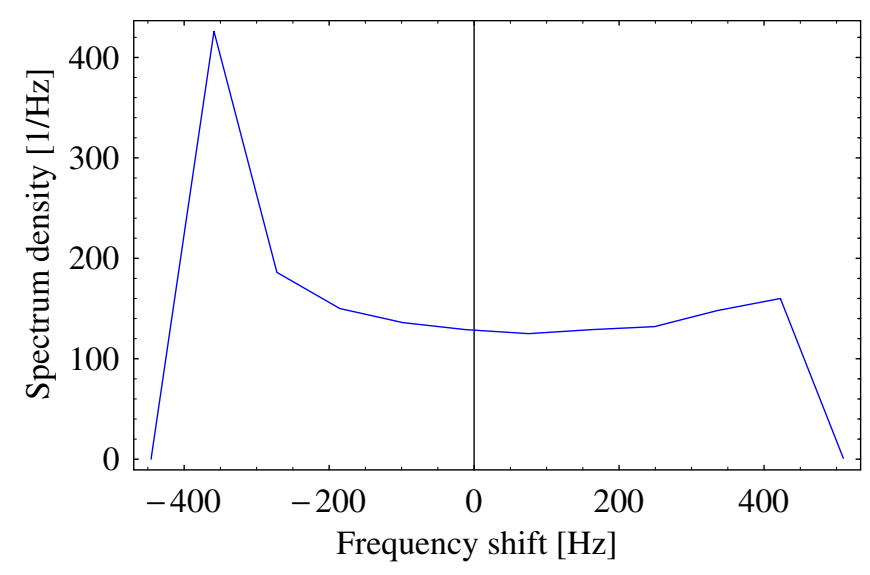

FIG. 7. (Color) The shape of the synchrotron sideband in the case where transients give dominant effect, parameters for LER at $1 \mathrm{~A}$ current.

In Fig. 6 we show the measured synchrotron frequency $\omega_{s}$ of a bunch centroid as a function of the beam current determined by two methods: the measurement of the first and the second sidebands of the rf frequency. $\omega_{s}$ measured by the second method shows linear dependence on the beam current and deviates from the measurements by the first method due to effect of the feedback.

The few comments ought to be added. Generally, the bunch centroid motion is a superposition of harmonics of the CB frequencies, including the second harmonics $2 \omega_{s, n}$. If the bunch-to-bunch frequency variation due to long gap transients is large, we can neglect bunch coupling caused by the longitudinal wake. In this case, all bunches have different frequencies and are approximately equal to $\omega_{s, n}$. The distribution of bunches over synchrotron frequencies calculated from the result depicted in the bottom plot of Fig. 2 for $1 \mathrm{~A}$ is shown in Fig. 7. It is similar to the actual profile of the sideband line in the spectrum observed in the LER. In the other extreme case, when the gap transients are small, the bunch frequencies are the frequencies of coupled-bunch modes and the set of these frequencies is the same for all bunches contrary to the shape of the spectrum for different bunches which still can be different because contributions of particular CB modes are not the same for different bunches.

For PEP-II LER, the spread of the CB modes is comparable with the variation of the synchrotron frequency along the train.

\section{B. Effect on luminosity}

Variation of the synchronous phase affects luminosity of the collider in two ways: First, through the induced variation of the bunch length $\sigma_{l}$ which, in its turn, changes luminosity $L$ due to the hour-glass effect. Second, the waist of the bunch shifts longitudinally and collision takes place at the point with larger $\beta$-function and, therefore, with larger transverse rms. The effect is shown in Fig. 8. The
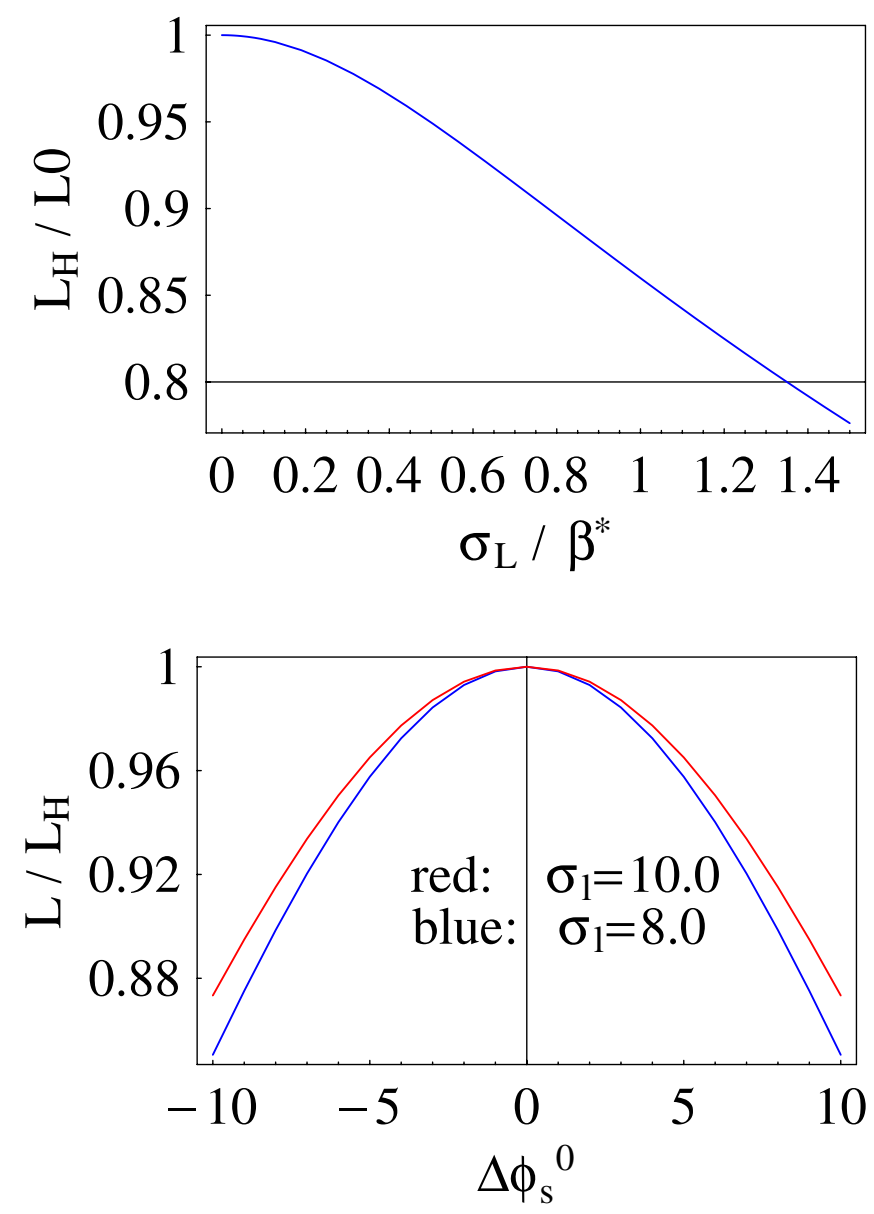

FIG. 8. (Color) Upper plot: Hour-glass effect due to $\sigma_{l}$ variation. Bottom plot: Variation of the luminosity with the synchronous phase error (due to gap transient) for $\sigma_{l}=10 \mathrm{~mm}$ (red), and $\sigma_{l}=8 \mathrm{~mm}$ (blue). Vertical beta function $\beta_{y}^{*}=1.0 \mathrm{~cm}$.

upper plot shows the hour-glass effect: variation of the luminosity $L_{H} / L_{0}$ normalized by the nominal luminosity $L_{0}$ of the pointlike bunches. The bottom plot shows additional drop of the luminosity $L / L_{H}$ with the shift of the synchronous phase of one of the bunches from the nominal phase. The luminosity is normalized on $L_{H}$. It is worth noting that, even if the rf phases of two beams are matched, the variation of the synchronous phase along the train remains and reduces the average luminosity. A special device such as dedicated harmonic cavity is needed to eliminate this adverse effect.

It is worth noting that the synchronous phase variation can be more important at large crossing angles suggested for the Super B factory [14]. The synchronous phase shift along the train can also be responsible for the variation of the tune along the train due to parasitic crossings.

\section{EFFECT OF THE RW WAKE ON TUNES}

As it is well known, the RW wake gives the dominant contribution to transverse dynamics, see Fig. 9. Cal- 


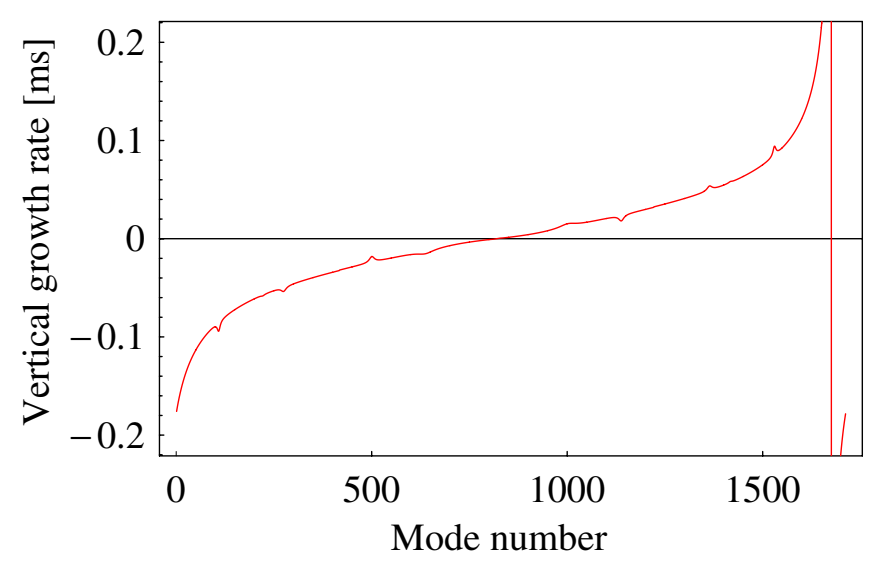

FIG. 9. (Color) Growth rate vs CB mode number for vertical oscillation at LER current of $3.5 \mathrm{~A}$.

culations of the corresponding tune variation of the transverse $\mathrm{CB}$ modes give $\delta Q_{x}=0.46 \times 10^{-2}$. Experiment (Fisher, gated tune measurements) gives variation along the train of the same order monotonic for $y$-plane and oscillatory in $x$-plane.

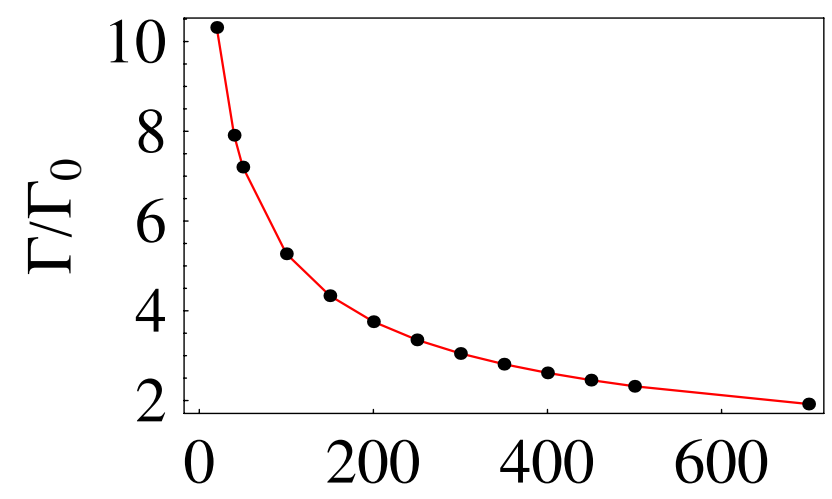

$\mathrm{n}_{\mathrm{b}}$

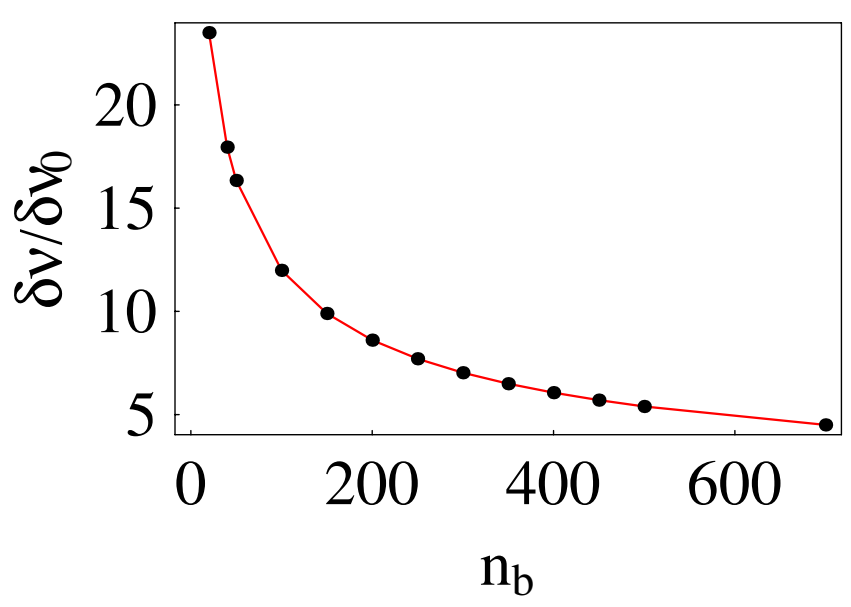

FIG. 10. (Color) Growth rate and the tune shift in vertical plane as function of the number of bunches for a fixed beam current and bunch spacing. PEP-II LER wake, $n_{b}=1712$.
This result deserves some comments. First of all, one can expect that all bunches are involved in $n_{B}$ CB oscillations. Although the amplitudes of different modes for each bunch can be different, the CB frequencies should be the same for all bunches, even if the wake is substantially reduced across the gap. However, transients due to e-cloud, parasitic crossings at IP combined with the variation of the synchronous phase shift along the train, and the quadrupolar wakefields can cause the bunch-to-bunch tune variation. Actually, if such a variation is larger than coupling between bunches, the CB modes are destroyed and bunches have different tunes. For large gaps, the tune depends not only on the beam current but also on the number of bunches [15]:

$$
\frac{d Q_{\perp}}{d I_{B}} \propto \frac{1}{n_{b}} \sqrt{\frac{R}{\sigma_{B}}}+\frac{\Gamma(1 / 4)}{\sqrt{1-Q_{\perp}}}+A .
$$

Here the first term is the single bunch effect, the second is multibunch effect, and the last term $A$ is the effect of the beam pipe asymmetry. The effect of the first two terms is shown in Fig. 10. The calculations were carried out directly solving the system of CB equation for $n$-bunches coupled by the RW wakefield.

The last term in Eq. (4) gives the dominant contribution for PEP-II. The very first measurements of the tune dependence on current gave the opposite slope in $x$ and $y$ planes, while for the round beam pipe they have to be both negative. Later measurements [16] at much higher currents confirmed such dependence (Fig. 11).

The effect has been explained $[15,17]$ as a result of the rectangular (more exactly, hexagonal) shape of the vacuum chamber in the arcs. The quadrupolar wakefield in this case depends on offsets of both the leading and trailing particles. The part of the wakes of all $n_{b} \simeq 1700$ bunches proportional to the offset of the trailing bunch are added up and the amplitude of the resulting wake depends on the time of diffusion of the wakefields through the beam pipe wall. The typical buildup time is about 200 turns. This part does not exist for a round beam pipe. Results of the calculation of the tune shift with current is shown in Table I, where we compare the tune variation without quadrupolar wake (upper box) and with it (the box in the middle) with the experimental tune variation used for the forward feedback in the control room. The calculations are based on real dimensions of the beam pipe but use a 1D estimate of the diffusion time. We also give for comparison the single bunch tune shift [18] where the slope of $d Q_{x, y} / d I$ has the same sign in both planes.

\section{A. Variation of the $\beta$-function and dispersion}

Variation of the tune with current leads, of course, to the variation of the Twiss parameters. For illustration, effect was calculated using the code MAD for $Q_{x}=38.518$. To simulate the effect of the wakefield on beam optics, we 

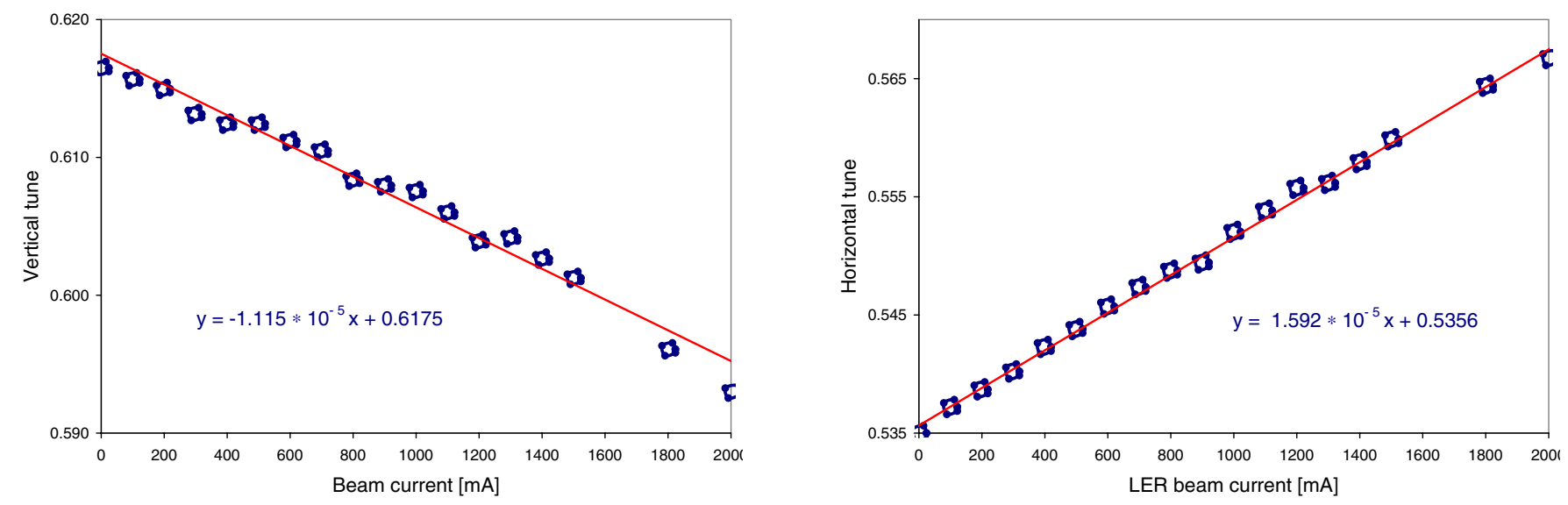

FIG. 11. (Color) Measured vertical (left) and horizontal (right) tune variation with LER current. Number of bunches is 1467.

TABLE I. Results of the calculations of the slope $d \nu / d I_{B}$ $(1 / \mathrm{mA})$, without and with the quadrupolar wakefield and comparison with the experiment. Compare with LER single bunch (Turner) results: $d \nu_{x} / d I_{\text {bunch }}=-0.00226(1 / \mathrm{mA})$, $d \nu_{y} / d I_{\text {bunch }}=-0.00131(1 / \mathrm{mA})$.

\begin{tabular}{lcc}
\hline \hline No $Q$-field & LER & HER \\
$x$ & $-0.036 \times 10^{-5}$ & $-0.015 \times 10^{-5}$ \\
$y$ & $-0.103 \times 10^{-5}$ & $-0.041 \times 10^{-5}$ \\
\hline With $Q$-field & LER & HER \\
$x$ & $1.620 \times 10^{-5}$ & $-0.800 \times 10^{-5}$ \\
$y$ & $-1.410 \times 10^{-5}$ & $-2.450 \times 10^{-5}$ \\
\hline Experiment & LER & HER \\
$x$ & $2.100 \times 10^{-5}$ & $2.000 \times 10^{-5}$ \\
$y$ & $-1.500 \times 10^{-5}$ & $-2.000 \times 10^{-5}$ \\
\hline \hline
\end{tabular}

added a small $\left(K_{1} \simeq 10^{-4}\right)$ quadrupole component to the main bends in the LER ring. This caused change of the tune by $\delta Q_{x} \simeq 10^{-3}$. One can expect such a change of the tune with current due to wakes at $I_{B}$ of couple amperes. The variation of the horizontal dispersion at IP, and the relative variation of the maximum of the beta function and dispersion in the ring is given below:

$$
\begin{gathered}
\frac{d D_{x}^{*}}{d Q_{x}}=4.10^{-2}, \quad \frac{1}{\beta_{x}^{\max }} \frac{d \beta_{x}^{\max }}{d Q_{x}}=1.62, \\
\frac{1}{D_{x}^{\max }} \frac{d D_{x}^{\max }}{d Q_{x}}=0.15 .
\end{gathered}
$$

\section{CONCLUSION}

The optical effects of the wakefields are needed to explain experimental observations at low impedance machines. At higher currents but below the coherent instability threshold, wakefield can affect the beam dynamics in the nonresonant way. We show that the bunch length and the synchronous phase of bunches can vary along the train of bunches. The variation is mostly defined by the beam loading to the fundamental mode of the rf cavities and depends on the detuning of the cavities. The bunch length can be different for a single bunch and the bunch length measured in a train of bunches. The relation between them may depend on the synchrotron tune. A detailed discussion on that is given in Appendix A. The measurements of the synchrotron frequency from the first and the second sidebands give different results and we show that the second sideband gives more reliable results. Variation of the synchronous phase along the train affects the luminosity on top of the well-known hour-glass effect. The resistive wall wakefields can change the tune and the effect depends on the number of the bunches in the bunch train and on the asymmetry of the beam pipe. Variation of the tune and the Twiss parameters along the train must be included in optimization of the machine main parameters. All these effects become serious issues for high current colliders. In particular, the effect of the variation of the synchronous phase along the train can be important for the design of the Super B factory with a large crossing angle.

\section{APPENDIX A: ON THE DEFINITION OF THE BUNCH LENGTH}

We summarize here basic formulas used in our calculations.

We denote the distance of the center of the rf bucket occupied by the $n$th bunch from the head of the train by $S_{n}$. In the case of equidistant bunches, $S_{n}=(n-1) s_{b}, n=$ $1,2, \ldots, n_{b}$, and $n=1$ is the head of the train. The $i$ th particle of the $n$th bunch on the $k$ th turn is located in the ring at the moment $t$ at $c t-S_{n}-\zeta_{n}+z_{n, i}-k C$, where $\zeta_{n}$ is related to the rf phase $\phi_{n}=\omega_{r f} \zeta_{n} / c$ of the $n$th bunch, and $z_{n, k}$ is the shift of particles from the bunch center. The shift is positive for offset toward the head of the train.

The longitudinal motion is described by a Hamiltonian $H=\sum H_{m}\left(p_{m, i}, z_{m, i}, s\right)$, where the canonical momentum $p_{m, i}=-\alpha \delta_{m, i}$ is defined in terms of the momentum com- 
paction $\alpha$ and the energy shift $\delta=\left(E-E_{0}\right) / E_{0}$, and

$$
\begin{aligned}
H_{n}= & \frac{p_{n, i}^{2}}{2}+\left(\frac{\omega_{s, 0}}{c}\right)^{2} \frac{z_{n, i}^{2}}{2}+\frac{\alpha}{E_{0} C}\left(e V_{\mathrm{rf}} \cos \left[\phi_{n}\right]-U_{l}\right) z_{n, i} \\
& +\lambda \int d z^{\prime} \rho_{n}\left(z^{\prime}, s\right) G\left[z^{\prime}-z_{n, i}\right] \\
& +\lambda \sum_{m, k} \int d z^{\prime} \rho_{m}\left(z^{\prime}, s\right) G\left[z^{\prime}-z_{n, i}+S_{n}-S_{m}+k C\right] .
\end{aligned}
$$

Here

$$
\lambda=\frac{\alpha N_{B} e^{2}}{E_{0} C}
$$

and we neglected the nonlinearities of the rf potential. Other notations are standard: $V_{\mathrm{rf}}$ is the rf voltage per turn, $\omega_{\mathrm{rf}}=h \omega_{0}, \omega_{0}=2 \pi c / C, U_{l}$ is the synchrotron radiation energy loss per turn per particle, $C=2 \pi R$ is circumference, $N_{B}$ is bunch population. The dimensionless function $G(z)$ is given by the two-particle longitudinal wake $W(z), G(z)=\int_{0}^{z} d z^{\prime} W\left(z^{\prime}\right)$, and prime in $W^{\prime}(z)$ means the derivative over the argument. Note that $G(z)=0$ for $z<0$, and $W(z)>0$ corresponds to the energy loss. The sum in Eq. (6) is over all bunches in the train preceding the bunch $n$ including previous turns. Finally, $\omega_{s 0}$ is the zerocurrent synchrotron frequency $\omega_{s 0}$,

$$
\left(\frac{\omega_{s, 0}}{\omega_{0}}\right)^{2}=\frac{\alpha e h V r f}{2 \pi E_{0}} \sin \phi_{n}
$$

For a bunch current below the threshold of the microwave instability, the density of the $n$th bunch in the phase plane can be written as $F_{n}(z, p, s)=F_{n}\left[z-Z_{n}(s), p\right]$ where $Z_{n}(s)$ describes oscillations of the bunch center. The bunch density $\rho_{n}$ then is

$$
\begin{aligned}
\rho_{n}(z, s)=\int d p F_{n}(z, p, s) & =f_{n}[z-Z(s)], \\
\int d z f_{n}(z, s) & =1 .
\end{aligned}
$$

It is convenient to use canonical variables $\hat{z}_{n, i}, \hat{p}_{n, i}$ describing motion in the frame oscillating with the bunch center,

$$
z_{n, i}=Z_{n}(s)+\hat{z}_{n, i}, \quad p_{n, i}=Z_{n}^{\prime}(s)+\hat{p}_{n, i} .
$$

The centroid trajectory $Z_{n}(s)$ is defined below.

The canonical transform to new variables can be done with the generating function $\Phi\left(\hat{p}_{n}, z_{n}, s\right)=\left(z_{n}-Z_{n}\right) \hat{p}_{n}+$ $Z_{n}^{\prime}(s) z_{n}$, where $Z_{n}^{\prime}=d Z_{n}(s) / d s$. The new Hamiltonian $\hat{H}\left(\hat{z}_{n}, \hat{p}_{n}\right)=H+\partial \Phi / \partial s$ takes the form

$$
\begin{aligned}
\hat{H}_{n}(\hat{z}, \hat{p})= & \frac{\hat{p}^{2}}{2}+\left(\frac{\omega_{s, 0}}{c}\right)^{2} \frac{\hat{z}^{2}}{2}+\left(\frac{\omega_{s, 0}}{c}\right)^{2} \hat{z} Z_{n}(s) \\
& +\frac{\alpha}{E_{0} C}\left(e V_{r f} \cos \left[\phi_{n}\right]-U_{l}\right) \hat{z}+Z_{n}^{\prime \prime}(s) \hat{z} \\
& +\lambda \int d z^{\prime} f_{n}\left(z^{\prime}\right) G\left[z^{\prime}-\hat{z}\right] \\
& +\lambda \sum_{m, k} \int d z^{\prime} f_{m}\left(z^{\prime}\right) G\left[k C+S_{n}-S_{m}\right. \\
& \left.+Z_{m}(s-k C)-Z_{n}(s)+z^{\prime}-\hat{z}\right] .
\end{aligned}
$$

Here the sum is over turns $k=0,1, \ldots$ and over all $M$ bunches in the ring $m=1,2, \ldots, M$ for $k \neq 0$ and for $m=$ $1,2, \ldots, n-1$ for $k=0$. Expanding the last term over $Z_{m}(s-k C)-Z_{n}(s)+z^{\prime}-\hat{z}_{n, i} \ll s_{b}$ and omitting terms independent of the canonical variables, we get

$$
\begin{aligned}
\hat{H}_{n}(\hat{z}, \hat{p})= & \frac{\hat{p}^{2}}{2}+\left(\frac{\hat{\omega}_{s}}{c}\right)^{2} \frac{\hat{z}^{2}}{2}+\lambda \int d z^{\prime} f_{n}\left(z^{\prime}\right) G\left[z^{\prime}-\hat{z}\right] \\
& +\lambda \hat{z} \int d z^{\prime} d z^{\prime \prime} f_{n}\left(z^{\prime}\right) f_{n}\left(z^{\prime \prime}\right) W\left(z^{\prime}-z^{\prime \prime}\right) .
\end{aligned}
$$

Here we used the definition of the bunch center $\int d z \rho_{n}(z) z=0$, notations

$$
\left(\frac{\hat{\omega}_{s}}{c}\right)^{2}=\left(\frac{\omega_{s, 0}}{c}\right)^{2}+\lambda \sum_{m, k} W^{\prime}\left[k C+S_{n}-S_{m}\right]
$$

equation for the rf phase

$$
\begin{aligned}
e V_{r f} \cos \left[\phi_{n}\right]= & U_{l}+N_{B} e^{2} \sum_{k, m} W\left[k C+S_{n}-S_{m}\right] \\
& +N_{B} e^{2} \int d z d z^{\prime} f_{n}(z) f_{n}\left(z^{\prime}\right) W\left(z^{\prime}-z\right),
\end{aligned}
$$

and defined $Z_{n}(s)$ as a solution of the coupled-bunch equations

$$
Z_{n}^{\prime \prime}(s)+\left(\frac{\hat{\omega}_{s}}{c}\right)^{2} Z_{n}(s)=\lambda \sum_{m, k} W^{\prime}\left[k C+S_{n}-S_{m}\right] Z_{m}(s-k C) .
$$

The last terms in Eqs. (A7) and (A9) are introduced to make location of the bunch centroid $\langle z\rangle=0$.

The rf phase $\phi_{n}$ is defined in Eq. (A9) by the total energy loss per turn. Note that the average contribution of the fundamental $\mathrm{rf}$ mode wake should not be included in Eq. (A9) because it is already taken into account as the beam loading contribution to the rf voltage.

For a nonuniform fill, the rf phases of different bunches are different. The transient effect is mostly given by fundamental mode of the rf cavities. Variation of $\phi_{n}$ along the train of bunches changes frequencies $\omega_{s 0}$, see Eq. (A2), affecting $\hat{\omega}_{s}$ of individual bunches additional to the effect of the wake of the last term in Eq. (A8). 
Equations (A8) and (A9) define frequencies of oscillations of bunch centers.

For the uniform distribution of the bunches around the ring, the solution of Eq. (A10) is given as a superposition of eigenmodes with the amplitudes $a_{\mu}$ :

$$
\begin{aligned}
Z_{n}(s) & =\sum_{\mu=0}^{M} a_{\mu} X_{n}^{\mu} e^{-i\left(\Omega_{\mu} / c\right)\left[s+(n-1) s_{b}\right]}, \\
X_{n}^{\mu} & =\frac{1}{\sqrt{M}} e^{2 \pi i\{(n-1) \mu] / M\}},
\end{aligned}
$$

where frequencies are given in terms of the longitudinal impedance per turn $Z(\omega)$,

$$
\begin{aligned}
\Omega_{\mu}^{2}-\omega_{s 0}^{2}= & i \lambda M \frac{\omega_{0}^{2}}{2 \pi} \sum_{p}\left\{\left(p M+\mu+\frac{\Omega_{\mu}}{\omega_{0}}\right)\right. \\
& \times Z\left[\left(p M+\mu+\frac{\Omega_{\mu}}{\omega_{0}}\right) \omega_{0}\right] \\
& \left.-p M Z\left[p M \omega_{0}\right]\right\} e^{-\left(p M+\mu+\Omega_{\mu} / \omega_{0}\right)^{2}\left[\left(2 \pi \sigma_{0}\right) / C\right]^{2}} .
\end{aligned}
$$

The Fokker-Plank equation with the Hamiltonian Eq. (A7) has the steady-state Haissinski solution,

$$
F(\hat{p}, \hat{z})=\frac{1}{|N|} e^{-\left[\hat{H}_{n}(\hat{z}, \hat{p})\right] / T},
$$

where $|N|$ is a normalization constant, and $f_{n}(\hat{z})=$ $\int d p F(\hat{p}, \hat{z})$. The normalization constant $|N|$ is defined by the condition $\int d z f_{n}(z)=1$. It is easy to check that the average $\langle\hat{z}\rangle$ is zero $\langle\hat{z}\rangle=\int d z z f_{n}(z)=0$.

The temperature $T$ should be defined to give the rms energy spread $\delta_{0}^{2}$. Hence, $\left\langle p^{2}\right\rangle=T=\left(\alpha \delta_{0}\right)^{2}$.

For currents below threshold of the microwave instability, parameter

$$
\Lambda=\frac{\lambda}{T}=\frac{N_{B} e^{2}}{E_{0} C \alpha \delta_{0}^{2}}
$$

is small. In the linear approximation,

$$
\begin{aligned}
f_{n}(\hat{z})= & \frac{1}{|N|} e^{-\left(\frac{\hat{\omega}_{s}}{c}\right) \frac{z^{2}}{2 T}\left\{1-\Lambda \int d z^{\prime} f_{n}\left(z^{\prime}\right) G\left[z^{\prime}-\hat{z}\right]\right.} \\
& \left.-\Lambda \hat{z} \int d z^{\prime} d z^{\prime \prime} f_{n}\left(z^{\prime}\right) f_{n}\left(z^{\prime \prime}\right) W\left(z^{\prime}-z^{\prime \prime}\right)\right\} .
\end{aligned}
$$

Simple calculations give the rms bunch length $\sigma$,

$$
\begin{aligned}
\sigma^{2}= & \int d z z^{2} f_{n}(z), \\
\sigma^{2}= & \sigma_{0}^{2}\left(\frac{\omega_{s 0}}{\hat{\omega}_{s}}\right)^{2}\left\{1+\frac{\Lambda}{2 \sqrt{2 \pi}} \int d x G\left[\frac{\omega_{s 0}}{\hat{\omega}_{s}} \sqrt{2} \sigma_{0} x\right]\right. \\
& \left.\times\left[1-x^{2}\right] e^{-\left(x^{2} / 2\right)}\right\}+o\left(\lambda^{2}\right) .
\end{aligned}
$$

In the linear approximation over $\Lambda, \omega_{s 0} / \hat{\omega}_{s}$ in the argument of $G$ can be replaced by one.

The frequency Eq. (A8) includes the effect of the longrange wakefield. If the multiturn wake can be neglected, then for a single bunch $\hat{\omega}_{s}=\omega_{s 0}$, and the single bunch rms $\sigma_{1}$ is

$$
\begin{aligned}
\sigma_{1}^{2}= & \sigma_{0}^{2}\left\{1+\frac{\Lambda}{2 \sqrt{2 \pi}} \int d x G\left[\sqrt{2} \sigma_{0} x\right]\left[1-x^{2}\right] e^{-\left(x^{2} / 2\right)}\right\} \\
& +o\left(\lambda^{2}\right) .
\end{aligned}
$$

Equations (A16) and (A17) show that in the linear approximation over the bunch current the rms of a bunch in a train and of a single bunch are different,

$$
\sigma_{1}^{2}=\left(\frac{\hat{\omega}_{s}}{\omega_{s, 0}}\right)^{2} \sigma^{2}
$$

The difference is due to the long-range wake.

As an example we calculated the $\mathrm{CB}$ frequencies for LER of PEP-II using our model of the LER PEP-II machine impedance and Eq. (A12). The frequencies of the dipole $\mathrm{CB}$ modes can be written as

$$
\Omega_{\mu}^{D}=\hat{\omega}_{s}+\frac{d \Omega_{\mu}^{D}}{d I_{B}} I_{B}, \quad \hat{\omega}_{s}=\omega_{s 0}-\frac{d \Omega_{0}}{d I_{B}} I_{B},
$$

where the slope for a dipole CB longitudinal mode is

$$
\begin{aligned}
\frac{d \Omega_{\mu}^{D}}{d I_{B}} & =\operatorname{Re}\left\{i \frac{\alpha \omega_{0}^{2}}{4 \pi \omega_{s 0} E_{0} / e} \sum_{p}(p M+\mu) Z\left[(p M+\mu) \omega_{0}\right]\right\}, \\
\frac{d \Omega_{0}}{d I_{B}} & =i \frac{\alpha \omega_{0}^{2}}{4 \pi \omega_{s 0} E_{0} / e} \sum_{p} p M Z[p M] .
\end{aligned}
$$

Calculations give

$$
\left\langle\frac{d \Omega_{\mu}^{D}}{d I_{B}}\right\rangle=1410\left(\frac{\mathrm{Hz}}{\mathrm{A}}\right), \quad \frac{d \Omega_{0}}{d I_{B}}=1417\left(\frac{\mathrm{Hz}}{\mathrm{A}}\right) .
$$

Two terms in $\Omega_{\mu}$ almost cancel in agreement with Fig. 6 at low currents. At high current the slope depends on the feedback and the nonlinear terms $o\left(I^{2}\right)$.

For the quadrupole modes the calculated slope of different modes is small and about constant for all modes,

$$
\Omega_{\mu}^{Q}=2 \hat{\omega}_{s}+\frac{d \Omega_{\mu}^{Q}}{d I_{B}} I_{B}, \quad \frac{d \Omega_{\mu}^{Q}}{d I_{B}} \simeq 246\left(\frac{\mathrm{Hz}}{\mathrm{A}}\right) .
$$

Therefore, the slope of $\Omega_{\mu}^{Q}$ is given with good accuracy by the first term $2 \hat{\omega}_{s}$ which allows one to define $\hat{\omega}_{s}$ from measurements of the slope of the second sideband in the beam spectrum.

With known $\frac{d \Omega_{0}}{d I_{B}}$, we can derive the rms length of a single bunch from measurements of the average bunch length $\sigma=\sigma_{0}+\left(d \sigma / d I_{B}\right) I_{B}$ in the train of bunches using Eq. (A18): 


$$
\begin{aligned}
\sigma_{1} & =\left(1-\frac{1}{\omega_{s 0}} \frac{d \Omega_{0}}{d I_{B}} I_{B}\right)\left(\sigma_{0}+\frac{d \sigma}{d I_{\text {bunch }}} I_{\text {bunch }}\right) \\
& \simeq \sigma_{0}+I_{\text {bunch }}\left(\frac{d \sigma}{d I_{\text {bunch }}}-M \frac{d \Omega_{0}}{d I_{B}} \frac{\sigma_{0}}{\omega_{s 0}}\right) .
\end{aligned}
$$

Equation (A23) is in agreement with Fig. 5 although the experimental slope has some uncertainty; see points in Fig. 5 indicating several measurements with the same current.

\section{ACKNOWLEDGMENTS}

We appreciate discussions with A. Chao and Y. Nosochkov. This work was supported by Department of Energy Contract No. DE-AC03-76SF00515.

[1] A. W. Chao, Physics of Collective Beam Instabilities in High Energy Accelerators (J. Wiley and Sons, Inc., New York, 1993).

[2] Yunhai Cai (private communication).

[3] J. M. Byrd, S. De Santis, J. Jacob, and V. Serriere, Phys. Rev. ST Accel. Beams 5, 092001 (2002).
[4] D. Alesini, A. Gallo, S. Guiducci, F. Marcellini, and M. Zobov, Phys. Rev. ST Accel. Beams 6, 074401 (2003).

[5] T. F. Gunzel, T. Perron, L. Farvacque, and J. L. Revol, Proceedings of 2005 PAC, Knoxville, Tennessee, p. 1217.

[6] V. Kiselev and V. Smaluk, Nucl. Instrum. Methods Phys. Res., Sect. A 525, 433 (2004).

[7] G. Arduni, C. Carli, and F. Zimmermann, Proceedings of EPAC 2004, Lucerne, Switzerland.

[8] E. Karantzoulis, V. Smaluk, and L. Tosi, Phys. Rev. ST Accel. Beams 6, 030703 (2003).

[9] Y. Luo, P. Cameron, A. Della Penna, Jr., J. Laster, R. Lee, A. Marusic, F. Pilat, T. Roser, D. Trbojevic, and J. Wei, Phys. Rev. ST Accel. Beams 8, 014001 (2005).

[10] Super-B-factory Workshop, SLAC, 2006.

[11] A. Fisher et al., Proceedings of 2005 PAC, Knoxville, Tennessee, 2005, p. 1934.

[12] D. Teytelman, Ph.D. thesis, Stanford University [SLAC-R633, 2003].

[13] S. Heifets et al., SLAC/AP-99, 1995.

[14] P. Raimondi, EPAC-2004-FRXBCH02.

[15] S. Heifets, SLAC-PUB-7985, 1998.

[16] U. Wienands (private communication).

[17] A. Chao, S. Heifets, and B. Zotter, Phys. Rev. ST Accel. Beams 5, 111001 (2002).

[18] J. Turner (private communication). 